Pacific Journal of Mathematics

ON A LINEAR FORM WHOSE DISTRIBUTION IS IDENTICAL
WITH THAT OF A MONOMIAL 


\title{
ON A LINEAR FORM WHOSE DISTRIBUTION IS IDENTICAL WITH THAT OF A MONOMIAL
}

\author{
R. G. LAHA AND E. LUKACS
}

Several authors studied identically distributed linear forms in independently and identically distributed random variables. J. Marcinkiewicz considered finite or infinite linear forms and assumed that the random variables have finite moments of all orders. He showed that the common distribution of the random variables is then the Normal distribution. Yu. V. Linnik obtained some deep results concerning identically distributed linear forms involving only a finite number of random variables. The authors have investigated in a separate paper the case where one of the linear forms contains infinitely many terms while the other is a monomial. They obtained a characterization of the normal distribution under the assumption that the second moment of the random variable is finite. In the present paper we investigate a similar problem and do not assume the existence of the second moment.

1. We prove the following theorem:

THEOREM. Let $\left\{X_{j}\right\}$ be a finite or denumerable sequence of independently and identically distributed nondegenerate random variables and let $\left\{a_{j}\right\}$ be a sequence of real numbers such that the sum $\sum_{j} a_{j} X_{j}$ exists ${ }^{1}$. Let $\alpha \neq 0$ be a real number such that

(i) the sum $\sum_{j} a_{j} X_{j}$ is distributed as $\alpha X_{1}$

$$
\sum_{j} \alpha_{j}^{2} \geqq \alpha^{2} \text {. }
$$

Then the common distribution of the $X_{j}$ is normal.

REMARK. The converse statement is evidently true provided that $\sum_{j} a_{j}=\alpha$ if the sum $\sum_{j} a_{j} X_{j}$ contains more than two terms or $\mathcal{E}\left(X_{j}\right)=0$ in case $\sum_{j} a_{j} X_{j}$ has only two terms.

In $\S 2$ we prove three lemmas, the third of these has some independent interest. In $\S 3$ the theorem is proved.

Received October 14, 1963, and in revised form March 10, 1964. The work of the first author was supported by the National Science Foundation under grant GP-96. The work of the second author was supported by the U.S. Air Force under grant AF-AFOSR-473-63.

1 We say that the infinite sum $\sum_{j} a_{j} X_{j}$ exists, if it converges almost everywhere. It is known (see Loève [3] pg. 251) that for a series of independent random variables the concepts of convergence almost everywhere and weak convergence are equivalent. 
2. Lemmas. We denote the common distribution of the random variable $X_{j}$ by $F(x)$ and write $f(t)$ for the corresponding characteristic function.

Lemma 1. Suppose that all the conditions of the theorem except (ii) are satisfied. Then $\sup _{j}\left|a_{j}\right|<|\alpha|$.

According to the assumptions we have

$$
\prod_{j} f\left(a_{j} t\right)=f(\alpha t) \text {. }
$$

We set $b_{j}=a_{j} / \alpha(j=1,2, \cdots)$ and obtain

$$
\prod_{j} f\left(b_{j} t\right)=f(t) \text {. }
$$

The lemma is proven if we show that $\left|b_{j}\right|<1$ for all $j$. First we note that if $\left|b_{j}\right|=1$ for at least one value of $j$, then $X_{j}$ has necessarily a degenerate distribution. We consider the case where $\left|b_{k}\right|>1$ for at least one value $k$. We see then from (2.2) that

$$
1 \geqq\left|f\left(b_{k} t\right)\right| \geqq|f(t)|
$$

which means

$$
1 \geqq|f(t)| \geqq\left|f\left(t / b_{k}\right)\right| \geqq\left|f\left(t / b_{k}^{2}\right)\right| \geqq \cdots \lim _{n \rightarrow \infty}\left|f\left(t / b_{k}^{n}\right)\right|=f(0)=1 .
$$

Therefore $|f(t)| \equiv 1$ and the distribution of $X_{j}$ is again degenerate. We conclude therefore that

$$
\left|b_{j}\right|<1 \quad(j=1,2 \cdots)
$$

Lemma 2. Suppose that all the conditions of the theorem, except. (ii), are satisfied then the function $f(t)$ has no real zeros.

We first remark that the existence of the infinite sum $\sum_{j} a_{j} X_{j}$ implies that the sequence of random variables $S_{N}=\sum_{j=N+1}^{\infty} a_{j} X_{j}$ converges to zero (as $N \rightarrow \infty$ ) with probability 1. It follows from the continuity theorem that

$$
\lim _{N \rightarrow \infty} \prod_{j=N+1}^{\infty} f\left(a_{j} t\right)=1
$$

uniformly in every finite $t$-interval.

Let $\varepsilon>0$ be an arbitrarily small number and let $T$ be a positive number. It follows then from (2.4) that there exists an $N_{0}=N_{0}(\varepsilon, T)$ such that for all $N \geqq N_{0}$ the inequality 


$$
\left|\prod_{j=N+1}^{\infty} f\left(b_{j} t\right)-1\right| \leqq \varepsilon
$$

holds uniformly for $|t| \leqq T$.

We give an indirect proof of Lemma 2. Suppose that the function $f(t)$ has real zeros and let $t_{0}$ be one of the zeros of $f(t)$ which is closest to the origin. Then

$$
\prod_{j} f\left(b_{j} t_{0}\right)=f\left(t_{0}\right)=0,
$$

so that either $f\left(b_{j} t_{0}\right)=0$ for at least one value of $j$ or the product is infinite and diverges to zero at the point $t=t_{0}$. The first case is impossible by virtue of (2.3) while the second contradicts the uniform convergence of the infinite product so that Lemma 2 is proven.

Lemma 3. Let $\left\{X_{j}\right\}$ be a finite or denumerable sequence of independently and identically distributed nondegenerate random variables and let $\left\{a_{j}\right\}$ be a sequence of real numbers such that the sum $\sum_{j} a_{j} X_{j}$ exists. Let $\alpha \neq 0$ be a real number such that $\sup _{j}\left|a_{j}\right|<|\alpha|$. Suppose that the sum $\sum_{j} a_{j} X_{j}$ has the same distribution as $\alpha X_{1}$, then the common distribution of each $X_{j}$ is infinitely divisible.

To prove Lemma 3 we write $(2.2)$ in the form ${ }^{2}$

$$
f(t)=f\left(b_{1} t\right) f\left(b_{2} t\right) \cdots f\left(b_{N} t\right) \Phi_{N}(t)
$$

where

$$
\Phi_{N}(t)=\ddot{\prod}_{j=N+1} f\left(b_{j} t\right)
$$

and where $N$ is so large that the inequality (2.5) holds. Using (2.6) we see that

$$
f(t)=\prod_{j=1}^{N} f\left(b_{j}^{2} t\right) \prod_{\substack{j, k=1 \\ j>k}}^{N}\left[f\left(b_{j} b_{k} t\right)\right]^{2}\left[\prod_{j=1}^{N} \Phi_{N}\left(b_{j}\right)\right] \cdot \Phi_{N}(t) .
$$

We repeat this process $n$ times and obtain

$$
\begin{aligned}
& f(t)=\left\{\prod_{j_{1}+\cdots+j_{N}=n}\left[f\left(b_{1}^{j_{1}} \cdots b_{N}^{j_{N}} t\right)\right]^{\left(n ; j_{1} \cdots j_{N}\right)}\right\} \\
& \cdot\left\{\prod_{k=1}^{n} \prod_{j_{1}+\cdots+j_{N}=n-k}\left[\Phi_{N}\left(b_{1}^{j_{1}} \cdots b_{N}^{j_{N}} t\right)\right]^{\left(n-k ; j_{1} \cdots j_{N}\right)}\right\} .
\end{aligned}
$$

Here all $j_{k} \geqq 0$ and $\left(m ; j_{1} \cdots j_{N}\right)=m ! / j_{1} ! \cdots j_{N}$ !. Formula (2.9) indicates that the random variable $X$, whose characteristic function is $f(t)$, is the sum of $k_{n}=N^{n}+N^{n-1}+\cdots+N^{2}+N+1$ independent random

${ }^{2}$ If the sequence $\left\{X_{j}\right\}$ is finite then $N$ is equal to the number of variables $X_{j}$ so that $\Phi_{N}(t) \equiv 1$. 
variables $X_{n, k}\left(k-1,2, \cdots, k_{n}\right)$, that is $X=\sum_{k=1}^{k_{n}} X_{n, k}$ for every $n$.

Such sequences of sums of independent random variables occur in the study of the central limit theorem, and we give next a few results which we wish to apply.

We say that the summands $X_{n, k}$ are uniformly asymptotically negligible (u.a.n), if $X_{n, k}$ converges in probability to zero, uniformly in $k$, as $n$ tends to infinity; this means that for any $\varepsilon>0$

$$
\lim _{n \rightarrow \infty} \max _{1 \leqq k \leqq k_{n}} P\left(\left|X_{n, k}\right| \geqq \varepsilon\right)=0 \text {. }
$$

It is known (see Loève [3] pg. 302) that condition (2.10) is equivalent to

$$
\lim _{n \rightarrow \infty} \max _{1 \leqq k \leqq k_{n}}\left|f_{n, k}(t)-1\right|=0
$$

uniformly in every finite $t$-interval.

Let $X_{n, k}\left(k=1,2, \cdots, k_{n}\right)$ be, for each $n$, a finite set of independent random variables and suppose that the $X_{n, k}$ are u.a.n. Then the limiting distribution (as $n$ tends to infinity) of the sums $\sum_{k=1}^{k}{ }_{n} X_{n, k}$ is infinitely divisible.

For the proof we refer the reader to Loève [3] (pg. 309).

We turn now to the proof of Lemma 3 and show that the factors of (2.9) satisfy condition (2.11).

Let $\varepsilon>0$ be an arbitrarily small number and $T>0$. We see from (2.5) and (2.7) that we can select a sufficiently large $N$ such that

$$
\left|\Phi_{N}(t)-1\right| \leqq \varepsilon
$$

uniformly in $|t| \leqq T$. Since $\left|b_{j}\right|<1$ we have

$$
\left|b_{1}^{j_{1}} \cdots b_{N}^{j_{N}} t\right|<T
$$

so that, according to (2.12),

$$
\left|\Phi_{N}\left(b_{1}^{j_{1}} \cdots b_{N}^{j_{N}} t\right)-1\right| \leqq \varepsilon
$$

uniformly in $|t| \leqq T$ for the chosen value of $N$.

We consider next a typical factor $f\left(b_{1}^{j_{1}} \cdots b_{N}^{j_{N}} t\right)$ of the product in the first brace of formula (2.9). Here $j_{1}+j_{2}+\cdots+j_{N}=n$ and $j_{k} \geqq 0$ so that at least one of the $j_{k}$ is positive. We, show now that it is possible to choose an $n_{0}=n_{0}(\varepsilon, T)$ such that for $n \geqq n_{0}$

$$
\gamma_{j_{1} \cdots j_{N}}(t)=\left|f\left(b_{1}^{j_{1}} \cdots b_{N}^{j_{N}} t\right)-1\right| \leqq \varepsilon
$$

uniformly in $|t| \leqq T$.

Clearly, 


$$
\begin{aligned}
\gamma_{j_{1} \cdots j_{N}}(t) \leqq & \left|\int_{|x| \geqq A}\left\{\exp \left[i b_{1}^{j_{1}} \cdots b_{N}^{j_{N}} t x\right]-1\right\} d F(x)\right| \\
& +\left|\int_{|x|<A}\left\{\exp \left[i b_{1}^{j_{1}} \cdots b_{N}^{j_{N}} t x\right]-1\right\} d F(x)\right| .
\end{aligned}
$$

We choose $A$ so large that

$$
\left|\int_{|x| \geqq A}\left\{\exp \left[i b_{1}^{j_{1}} \cdots b_{N}^{j_{N}} t x\right]-1\right\} d F(x)\right| \leqq 2 \int_{|x| \geqq A} d F(x) \leqq \frac{\varepsilon}{2} .
$$

We note that

$$
\left|\int_{|x|<A}\left\{\exp \left[i b_{1}^{j_{1}} \cdots b_{n}^{j_{N}} t x\right]-1\right\} d F(x)\right| \leqq\left|b_{1}^{j_{1}} \cdots b_{N}^{j_{N}}\right| T A .
$$

We select now an $n^{*}=n^{*}\left(j_{1}, \cdots, j_{N} ; T, \varepsilon\right)$ so large that for $n \geqq n^{*}$ the inequality

$$
\left|b_{1}^{j_{1}} \cdots b_{N}^{j_{N}}\right| T A \leqq \frac{\varepsilon}{2}
$$

holds. This is possible in view of (2.3). There are altogether $N^{n}$ terms of the form $f\left(b_{1}^{j_{1}} \cdots b_{N}^{j_{N}} t\right)$ in (2.14) and we choose

$$
n_{0}=n_{0}(\varepsilon, T)=\max _{j_{1} \cdots j_{N}} n^{*}\left(j_{1}, \cdots, j_{N} ; T, \varepsilon\right) ;
$$

then (2.14) follows from (2.16), (2.17), (2.18) and (2.19).

We see therefore that the set of independent random variables $X_{n, k}$ satisfies the u.a.n. condition (2.11). Therefore the distribution of $X$ is infinitely divisible and Lemma 3 is proven.

Since $f(t)$ is an infinitely divisible characteristic function, it admits the Lévy-Khinchine representation

$$
\begin{aligned}
\ln f(t)=i \alpha t-\beta t^{2} / 2 & +\int_{-\infty}^{-0}\left(e^{i t x}-1-\frac{i t x}{1+x^{2}}\right) \frac{1+x^{2}}{x^{2}} d G(x) \\
& +\int_{+0}^{\infty}\left(e^{i t x}-1-\frac{i t x}{1+x^{2}}\right) \frac{1+x^{2}}{x^{2}} d G(x)
\end{aligned}
$$

where $\alpha$ and $\beta$ are real numbers, $\beta \geqq 0$, and where $G(x)$ is a nondecreasing, right-continuous function such that $G(-\infty)=0$ and $G(+\infty)$ $=K<\infty$. Let now $f(t)$ be the characteristic function of an infinitely divisible symmetric distribution, so that $f(t)=f(-t)$. In this case one sees after some elementary transformations of the integrals in (2.20) that

$$
G(x)+G(-x-0)=C
$$

for all $x \neq 0$. Using $(2.20)$ and (2.21) we see that the characteristic 
function of a symmetric infinitely divisible distribution admits the representation

$$
\ln f(t)=-\beta t^{2} / 2+\int_{+0}^{+\infty}(\cos t x-1) \frac{1+x^{2}}{x^{2}} d H(x)
$$

where

$$
H(x)= \begin{cases}2 G(x)-C & \text { for } x>0 \\ 0 & \text { for } x<0 .\end{cases}
$$

Thus $H(x)$ is a non decreasing, right-continuous, bounded function and $H(x)$ and $G(x)$ determine each other uniquely.

3. Proof of the theorem. We introduce the function

$$
g(t)=f(t) f(-t)
$$

and conclude from (2.2) that the relation

$$
\prod_{j} g\left(b_{j} t\right)=g(t)
$$

holds for all real $t$. Here $g(t)$ is the characteristic function of a symmetric distribution and is therefore a real and even function. It is no restriction to assume that

$$
0 \leqq b_{j}<1
$$$$
(j=1,2, \cdots)
$$

where

$$
\sum_{j=1}^{\infty} b_{j}^{2} \geqq 1 .
$$

According to (2.22) we have then the representation

$$
\ln g(t)=-\beta t^{2} / 2+\int_{+0}^{\infty}(\cos t x-1) \frac{1+x^{2}}{x^{2}} d H(x)
$$

where $\beta \geqq 0$ and where $H(x)$ is a nondecreasing, right-continuous and bounded function. We use (3.4) and (3.3b) and obtain from (3.2) the relation

$$
\begin{aligned}
& \sum_{j=1}^{\infty} \int_{+0}^{\infty}\left(\cos b_{j} t x-1\right) \frac{1+x^{2}}{x^{2}} d H(x) \\
& \quad=K \frac{t^{2}}{2}+\int_{+0}^{\infty}(\cos t x-1) \frac{1+x^{2}}{x^{2}} d H(x) .
\end{aligned}
$$

where

$$
K=\beta\left[\sum_{j=1}^{\infty} b_{j}^{2}-1\right] \geqq 0 \text {. }
$$


We define the sequence $\left\{\psi_{\nu}(t)\right\}$ by

$$
\psi_{\nu}(t)=\sum_{j=1}^{\nu} \int_{+0}^{\infty}\left(\cos b_{j} t x-1\right) \frac{1+x^{2}}{x^{2}} d H(x)
$$

so that

$$
\lim _{\nu \rightarrow \infty} \psi_{\nu}(t)=\psi(t)=K \frac{t^{2}}{2}+\int_{+0}^{\infty}(\cos t x-1) \frac{1+x^{2}}{x^{2}} d H(x)
$$

for every real $t$.

Since $\psi(t)$ is the characteristic function of an infinitely divisible distribution it follows that $K \leqq 0$, so that we conclude from assumption (ii) that $K=0$ and $\sum_{j=1}^{\infty} b_{j}^{2}=1$.

By a change of the variable of integration in (3.6) we obtain

$$
\psi_{\nu}(t)=\int_{+0}^{\infty}(\cos t x-1) \frac{1+x^{2}}{x^{2}}\left[\sum_{j=1}^{\nu} \frac{b_{j}^{2}+x^{2}}{1+x^{2}} d H\left(x / b_{j}\right)\right] .
$$

We write

$$
H_{\nu}(x)= \begin{cases}\int_{+0}^{x}\left[\sum_{j=1}^{\nu} \frac{b_{j}^{2}+y^{2}}{1+y^{2}} d H\left(y / b_{j}\right)\right] & \text { for } x>0 \\ 0 & \text { for } x<0 .\end{cases}
$$

Therefore we have, for every $\nu$,

$$
\psi_{\nu}(t)=\int_{+0}^{+\infty}(\cos t x-1) \frac{1+x^{2}}{x^{2}} d H_{\nu}(x) .
$$

It follows then from (3.7) and (3.8) that

$$
\lim _{\nu \rightarrow \infty} H_{\nu}(x)=H(x)
$$

for every $x$ which is a continuity point of $H(x)$. The proof is carried in the same way in which the convergence theorem is proven (see Loève [3] pp. 300-301).

In view of $(3.3 \mathrm{a})$ we have

$$
\frac{b_{j}^{2}+y^{2}}{1+y^{2}} \geqq b_{j}^{2} \quad(j=1,2, \cdots)
$$

so that we conclude from (3.8) that

$$
H_{\nu}(x) \geqq \sum_{j=1}^{\nu} b_{j}^{2} H\left(\frac{x}{b_{j}}\right)
$$

for all $\nu$.

It follows from (3.10) and (3.11) that 


$$
H(x)=\lim _{\nu \rightarrow \infty} H_{\nu}(x) \geqq \sum_{j=1}^{\infty} b_{j}^{2} H\left(\frac{x}{b_{j}}\right)
$$

for all $x>0$ which are continuity points of $H(x)$.

Using equation $(3.3 \mathrm{~b})$ we obtain

$$
\sum_{j=1}^{\infty} b_{j}^{2}\left[H(x)-H\left(\frac{x}{b_{j}}\right)\right] \geqq 0 .
$$

Since $H(x)$ is a nondecreasing function, we see from (3.3a) that

$$
H(x) \leqq H\left(\frac{x}{b_{j}}\right) .
$$

It follows from (3.12) and (3.13) that

$$
H(x)=H\left(\frac{x}{b_{j}}\right)
$$

for every $x>0$ which is a continuity point of $H(x)$. Therefore

$$
H(x)=H(+\infty)=C
$$

for $x>0$. We now turn to equation (3.4) and get

$$
\ln g(t)=-\beta t^{2} / 2 \text {. }
$$

The statement of the theorem is an immediate consequence of (3.1) and of Cramér's theorem.

\section{REFERENCES}

1. R. G. Laha-E. Lukacs, On linear forms and stochastic integrals, (to be published). 2. Yu. V. Linnik, Linear forms and statistical criteria I, II, Ukrainski Mat. Zurnal 5 (1953), 207-243 and 247-290. [English translation in Selected Translations in Mathematical Statistics and Probability vol. 3, pp. 1-90, American Math. Soc., Providence, R.I. (1962)].

3. M. Loève, Probability Theory (third edition), D. Van Nostrand, New York (1963).

4. J. Marcinkiewicz, Sur une propriété de la loi de Gauss, Math. Zeitschr. 44 (1949), 612-618.

The Catholic University of America 


\section{PACIFIC JOURNAL OF MATHEMATICS}

\section{EDITORS}

H. Samelson

Stanford University

Stanford, California

R. M. Blumenthal

University of Washington

Seattle, Washington 98105
J. Dugundu

University of Southern California Los Angeles, California 90007

*Richard Arens

University of California

Los Angeles, California 90024

\section{ASSOCIATE EDITORS}
E. F. BECKENBACH
B. H. NeumanN
F. WOLF
K. YOSIDA

\section{SUPPORTING INSTITUTIONS}

UNIVERSITY OF BRITISH COLUMBIA CALIFORNIA INSTITUTE OF TECHNOLOGY UNIVERSITY OF CALIFORNIA MONTANA STATE UNIVERSITY

UNIVERSITY OF NEVADA

NEW MEXICO STATE UNIVERSITY

OREGON STATE UNIVERSITY

UNIVERSITY OF OREGON

OSAKA UNIVERSITY

UNIVERSITY OF SOUTHERN CALIFORNIA
STANFORD UNIVERSITY

UNIVERSITY OF TOKYO

UNIVERSITY OF UTAH

WASHINGTON STATE UNIVERSITY

UNIVERSITY OF WASHINGTON

AMERICAN MATHEMATICAL SOCIETY CALIFORNIA RESEARCH CORPORATION SPACE TECHNOLOGY LABORATORIES NAVAL ORDNANCE TEST STATION 


\section{Pacific Journal of Mathematics}

\section{Vol. 15, No. $1 \quad$ September, 1965}

Donald Charles Benson, Unimodular solutions of infinite systems of linear

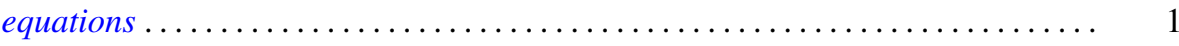

Richard Earl Block, Transitive groups of collineations on certain designs . . . . . . 13

Barry William Boehm, Existence of best rational Tchebycheff approximations .... . 19

Joseph Patrick Brannen, A note on Hausdorff's summation methods . . . . . . . . . . 29

Dennison Robert Brown, Topological semilattices on the two-cell ............ 35

Peter Southcott Bullen, Some inequalities for symmetric means . . . . . . . . . . 47

David Geoffrey Cantor, On arithmetic properties of coefficients of rational

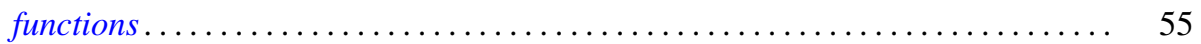

Luther Elic Claborn, Dedekind domains and rings of quotients . . . . . . . . . 59

Allan Clark, Homotopy commutativity and the Moore spectral sequence ........ 65

Allen Devinatz, The asymptotic nature of the solutions of certain linear systems of

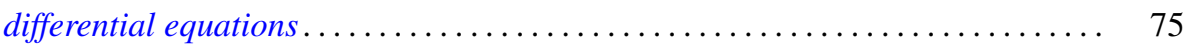

Robert E. Edwards, Approximation by convolutions ................... 85

Theodore William Gamelin, Decomposition theorems for Fredholm operators . . . . . 97

Edmond E. Granirer, On the invariant mean on topological semigroups and on

topological groups .................................. 107

Noel Justin Hicks, Closed vector fields . . . . . . . . . . . . . . . . . . . 141

Charles Ray Hobby and Ronald Pyke, Doubly stochastic operators obtained from

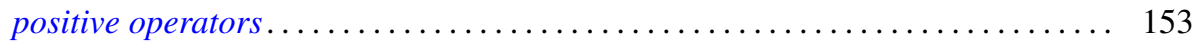

Robert Franklin Jolly, Concerning periodic subadditive functions . . . . . . . . . 159

Tosio Kato, Wave operators and unitary equivalence . . . . . . . . . . . . . . 171

Paul Katz and Ernst Gabor Straus, Infinite sums in algebraic structures . . . . . . . 181

Herbert Frederick Kreimer, Jr., On an extension of the Picard-Vessiot theory ...... 191

Radha Govinda Laha and Eugene Lukacs, On a linear form whose distribution is

identical with that of a monomial ......................... 207

Donald A. Ludwig, Singularities of superpositions of distributions . . . . . . . . . 215

Albert W. Marshall and Ingram Olkin, Norms and inequalities for condition

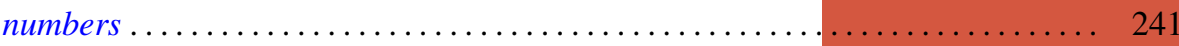

Horace Yomishi Mochizuki, Finitistic global dimension for rings . . . . . . . . . . 249

Robert Harvey Oehmke and Reuben Sandler, The collineation groups of division

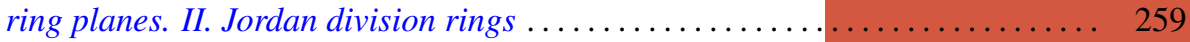

George H. Orland, On non-convex polyhedral surfaces in $E^{3} \ldots \ldots \ldots \ldots \ldots \ldots \ldots 267$

Theodore G. Ostrom, Collineation groups of semi-translation planes . . . . . . . . 273

Arthur Argyle Sagle, On anti-commutative algebras and general Lie triple

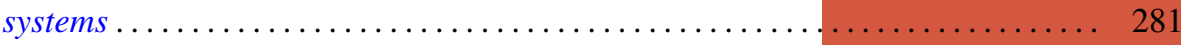

Laurent Siebenmann, A characterization of free projective planes . . . . . . . . . 293

Edward Silverman, Simple areas.................................. 299

James McLean Sloss, Chebyshev approximation to zero .................. 305

Robert S. Strichartz, Isometric isomorphisms of measure algebras . . . . . . . . . 315

Richard Joseph Turyn, Character sums and difference sets . . . . . . . . . . . . 319

L. E. Ward, Concerning Koch's theorem on the existence of arcs . . . . . . . . . . 347

Israel Zuckerman, A new measure of a partial differential field extension ......... 357 